\title{
ANALISE ESPACIAL DA CONCENTRAÇÃO DE NITROGENIO E FÓSFORO NO TECIDO FOLIAR DO CAFEEIRO CONILON
}

\author{
Abel Souza da Fonseca ${ }^{1}$ \\ Mariana Lima de Jesus ${ }^{2}$ \\ Tiago Machado da Silva ${ }^{3}$ \\ Samuel de Assis Silva ${ }^{4}$ \\ Julião Soares de Souza Lima ${ }^{5}$
}

Resumo: O conhecimento da variabilidade dos fatores que afetam a produtividade é importante para o manejo da cultura, planejamento de esquemas de amostragem e gerenciamento de práticas agrícolas. $O$ estudo foi realizado com a coleta de folhas recém-maduras para determinação dos teores de $P$ e $K$. Fez-se a análise de estatística descritiva e geoestatística dos teores foliares. Definida a dependência espacial, foram confeccionados mapas temáticos por meio da krigagem ordinária. Ambos os nutrientes apresentaram dependência espacial na área, sendo que a geoestatística permitiu mapear a variabilidade espacial e identificar diferentes zonas de manejo na área. Os resultados mostram deficiência de $N$ e $P$ na área, sendo que essa deficiência foi menor para o $P$. Os mapas identificam as regiões na área que devem receber maior atenção no momento da aplicação de adubos nitrogenados e fosfatados.

Palavras-chave: Estado nutricional; Geoestatística; Variabilidade espacial; Coffea canephora.

\footnotetext{
${ }^{1}$ Doutorando em Produção Vegetal/Centro de Ciências Agrárias e Engenharias/Cachoeiro de Itapemirim- ES, Brasil. E-mail: abelsouzafonseca@gmail.com.

2 Centro de Ciências Agrárias e Engenharias/Universidade Federal do Espírito Santo, Brasil. E-mail: marianaldejesus@gmail.com.

${ }^{3}$ Agronomia/Centro de Ciências Agrárias e Engenharias/Universidade Federal do Espírito Santo, Alegre- ES, Brasil. E-mail: tiagomachadao@hotmail.com.

${ }^{4}$ Universidade Federal do Espírito Santo/ Alegre- ES, Brasil. E-mail: sassilva@gmail.com.

5 Departamento de Engenharia Rural/Centro de Ciências Agrárias e Engenharias/Universidade Federal do Espírito Santo, Brasil. E-mail: limajss@yahoo.com.br.
} 